\title{
Comparison of different approaches to describe the thermotropic volume phase transition of smart microgels
}

\author{
Simon Friesen ${ }^{1} \cdot$ Yvonne Hannappel $^{1} \cdot$ Sergej Kakorin $^{1} \cdot$ Thomas Hellweg $^{1}$ (C)
}

Received: 19 November 2021 / Revised: 12 January 2022 / Accepted: 13 January 2022 / Published online: 21 February 2022

(c) The Author(s) 2022

\begin{abstract}
The description of gel swelling by Flory and Rehner using the original Flory-Huggins interaction parameter for the polymer-solvent interaction cannot be applied to most smart microgels. Here, we compare descriptions of the swelling curves of such microgels using series expansions of the Flory-Huggins parameter $\chi$ with the results of Hill-like equation for $\chi$. We study $N$-isopropyl-acrylamide particles at different concentrations of the cross-linker $N, N$-methylenebisacrylamide. The hydrodynamic radius $R_{\mathrm{H}}$ of the microgel particles is determined using photon correlation spectroscopy. The fits with the series expansion of $\chi$ nicely follow the experimental data. However, already with the first-order series expansion, the computed $\Theta$ temperatures are not physically reasonable. Moreover, the physical meaning of the parameters of the series expansion is not clear. The Hill-like equation, which we recently introduced, yields a good description of all measured microgel swelling curves and provides physically meaningful parameters. For instance, the Hill parameter $v$ corresponds to the number of water molecules per network chain cooperatively leaving the chain at the volume phase transition.
\end{abstract}

Keywords Flory-Rehner theory $\cdot$ Microgel $\cdot$ Hill cooperativity $\cdot$ Flory-Huggins parameter $\cdot$ Swelling behavior $\cdot$ NIPAM

\section{Introduction}

Microgels are colloidal particles made of a cross-linked polymer network that is permeated by a solvent. A special class of microgels are thermoresponsive in which the polymer network is permeated by water [1]. Thermoresponsive microgels display a volume phase transition temperature (VPTT) at which they abruptly change their degree of swelling $[2,3]$. Such so-called smart microgels are promising in regard to applications and therefore intensive research on these thermoresponsive colloids has been carried out in the last decades [1-11]. For instance, smart microgels are used as drug delivery systems [12-14], as carriers for

Thomas Hellweg

thomas.hellweg@uni-bielefeld.de

Simon Friesen

simon.friesen@uni-bielefeld.de

Yvonne Hannappel

yvonne.hannappel@uni-bielefeld.de

Sergej Kakorin

sergej.kakorin@uni-bielefeld.de

1 Department of Chemistry, Physical and Biophysical Chemistry, Bielefeld University, Universitätsstr. 25, Bielefeld 33615, Nordrhein-Westfalen, Germany enzymes[15, 16], as responsive surface coatings [17], or in catalysis [18-20]. The most frequently studied thermoresponsive microgel is based on the polymer poly $(N$-isopropylacrylamide) (p(NIPAM)) which in water exhibits a volume phase transition (VPT) at about $33{ }^{\circ} \mathrm{C}[2,3]$. The dramatic volume variation of polymeric microgel particles in response to temperature change is one of the central topics in polymer science [21]. The temperature-dependent swelling behavior is usually described by the Flory-Rehner theory [22-24]. The theory is based on the Flory-Huggins theory which is combined with the affine network model of elasticity. The most important factor describing the volume phase transition is the Flory-Huggins parameter $\chi$, which describes the change in enthalpy and entropy during the mixing process [25]. The interaction parameter describes the change in free energy per solvent molecule when a polymer-polymer and solvent-solvent contact is replaced by a polymer-solvent contact [23, 26-29]. The simple forms of the equations for $\chi$, like the original approach of Flory and Huggins for the polymer-solvent interaction, do not adequately describe the swelling behavior of most microgels [30].

Erman and Flory therefore proposed a series expansion because they assumed that higher-order interactions must be considered [30]. However, the original approach and the 
series expansion neglect that the cross-linking of the polymers leads to a cooperativity of the volume phase transition since all chains are mechanically coupled.

In the present paper, the parameter of the Flory-Rehner equation has been successfully modeled, either by a series expansion or by a more advanced Hill-like equation which describes the microgel collapse as a cooperative thermotropic transition $[31,32]$. It is physically clear that in the cross-linked networks of $\mathrm{p}$ (NIPAM) particles with the crosslinker $N, N$-methylenebisacrylamide (BIS) studied here, the polymeric chains are mechanically connected. Therefore, the thermotropic transition is expected to be a cooperative process. Due to this the Hill-like equation yields a very good description of all microgel swelling curves measured in the present work. It also provides a physically meaningful parameter $v$, the so-called Hill parameter [32]. The experimentally observed linear decrease in $v$ with increasing concentration of the cross-linker BIS in the microgel particles suggests that the Hill parameter $v$ is related to the mean number of water molecules per network chain cooperatively leaving the chain at the volume phase transition. In this paper, the different approaches are compared and the physical meaning of the parameters is discussed on the basis of typical p(NIPAM) microgel swelling curves.

\section{Theory}

\section{Flory-Rehner theory}

The Flory-Rehner theory represents an extension of the Flory-Huggins theory, which can be used to describe the temperature dependence of the swelling behavior of thermoresponsive microgels. This theory takes into account not only the solubility behavior but also the deformation of the elastic polymer network. The thermodynamic equilibrium of the gel is reached when the chemical potential of the solvent $\mu_{S}$ inside and outside the gel is equal.

$\mu_{S}^{\text {in }}=\mu_{S}^{\text {out }}$.

With the molar volume of the solvent $v_{S}$, the change of the chemical potential is related to the osmotic pressure $\Pi$ :

$\Pi=-\frac{\mu_{S}^{\text {in }}-\mu_{S}^{\text {out }}}{v_{S}}=\frac{N_{A}}{v_{S}} \cdot \frac{\partial \Delta F}{\partial N_{S}}=0$

where $N_{A}$ is the Avogadro constant, $N_{S}$ is the number of solvent molecules, and $\Delta F$ is the change in Helmholtz free energy. At this point, it should be mentioned that the free energy is equivalent to the free enthalpy because we assume an in-compressible lattice therefore the total volume theoretically does not change. For uncharged polymer networks, the osmotic pressure is composed of the osmotic pressure
$\Pi_{m i x}$ that occurs during mixing and the elastic contribution to the osmotic pressure $\Pi_{e l}$ that occurs related to deformation of the polymer network [23].

$\Pi=\Pi_{m i x}+\Pi_{e l}=0$.

The osmotic pressure that occurs during mixing is derived from Flory-Huggins theory. The free energy $\Delta F_{\text {mix }}$ being released during the mixing process of a polymer in a solvent is given by the change of enthalpy $\Delta H_{m i x}$, temperature $T$ and the change of entropy $\Delta S_{m i x}$ at constant pressure and volume.

$\Delta F_{\text {mix }}=\Delta H_{\text {mix }}-T \Delta S_{\text {mix }}$.

To calculate the change in free energy $\Delta F_{m i x}$, a fluid lattice is used as a model. With the number of possible arrangements $\Omega=\frac{\left(N_{S}+N_{M}\right) !}{N_{S} ! N_{M} !}$ and the Boltzmann constant $\mathrm{k}_{\mathrm{B}}$, the entropy change $\Delta S_{m i x}$ is given by:

$\Delta S_{m i x}=\mathrm{k}_{\mathrm{B}} \ln \left[\frac{\left(N_{S}+N_{M}\right) !}{N_{S} ! N_{M} !}\right]$

where $N_{S}$ is the number of lattice sites occupied by solvent molecules and $N_{M}$ is the number of lattice sites occupied by monomer units of the polymer. Each solvent molecule and each monomer unit of the polymer occupy one lattice site. It is assumed that all lattice sites have the same volume. Accordingly, the theory does not take into account the difference in volume of monomer units and solvent molecules. Using Stirling's approximation $\ln N ! \approx N \ln N-N$ and introducing the volume fractions $\phi_{S}=\frac{N_{S}}{N_{S}+N_{M}}$ and $\phi=\frac{N_{M}}{N_{S}+N_{M}}$, the entropy change $\Delta S_{m i x}$ is given by:

$\Delta S_{m i x}=-\mathrm{k}_{\mathrm{B}}\left[N_{S} \ln \phi_{S}+N_{M} \ln \phi\right]$

where $\phi_{S}$ is the volume fraction of the solvent and $\phi$ is the volume fraction of the polymer. From the change of the interaction energy $\Delta \varepsilon$ occurring during the formation of a polymer-solvent contact and from the number of these contacts $p$, the change of the enthalpy $\Delta H_{\text {mix }}$ can be described by the van Laar expression [22]:

$\Delta H_{\text {mix }}=\Delta \varepsilon p=\Delta \varepsilon N_{S} z \phi$

where $z$ is the coordination number of the liquid lattice. At this point the interaction parameter $\chi=\frac{\Delta \varepsilon z}{\mathrm{k}_{\mathrm{B}} T}$ is introduced. This represents a dimensionless quantity characterizing the interaction energy of a solvent molecule [22]. The interaction parameter is crucial for the volume phase transition, this parameter will be discussed in more detail in the subsection Interaction Parameter. The change in enthalpy $\Delta H_{m i x}$ can now be described by the interaction parameter:

$\Delta H_{m i x}=\mathrm{k}_{\mathrm{B}} T \chi N_{S} \phi$.

Using Eqs. (8) and (6), the osmotic pressure $\Pi_{m i x}$ is given by: 
$\Pi_{m i x}=-\frac{N_{A} \mathrm{k}_{\mathrm{B}} T}{v_{S}}\left[\ln (1-\phi)+\phi+\chi \phi^{2}\right]$.

For the elastic contribution to the osmotic pressure $\Pi_{e l}$, Flory and Rehner assumed the ideal case where each polymer chain length changes linearly with macroscopic deformation [22]. The entropy change $\Delta S_{e l}$ that occurs with this isotropic deformation is described by the number of polymer chains in the polymer network $N_{C}$, the Boltzmann constant $\mathrm{k}_{\mathrm{B}}$, and the linear swelling ratio $\alpha$ :

$\Delta S_{e l}=\frac{3 N_{C} \mathrm{k}_{\mathrm{B}}}{2} \cdot\left(\ln \alpha-\alpha^{2}+1\right)$

where the linear swelling ratio is defined by the volume of the microgel $V$ at a given state and the volume of the microgel in the reference state $V_{0}$. Usually, the collapsed state at high temperatures is chosen as the reference state. The swelling ratio $\alpha$ is often also described by the polymer volume fraction $\phi$ or by the hydrodynamic radius $R_{H}$ :

$\alpha \equiv\left(\frac{V}{V_{0}}\right)^{1 / 3}=\left(\frac{\phi_{0}}{\phi}\right)^{1 / 3}=\frac{R_{\mathrm{H}}}{R_{\mathrm{H}, 0}}$

where $\phi_{0}$ and $R_{\mathrm{H}, 0}$ are the values of the microgel in the reference state and $\phi$ and $R_{\mathrm{H}}$ are the values at a given state. From the change in entropy $\Delta S_{e l}$, the change in free energy $\Delta F_{e l}=-T \Delta S_{e l}$ and osmotic pressure $\Pi_{e l}$ can be calculated:

$\Pi_{e l}=\frac{N_{C} \mathrm{k}_{\mathrm{B}} T}{V_{0}} \cdot\left[\frac{\phi}{2 \phi_{0}}-\left(\frac{\phi}{\phi_{0}}\right)^{1 / 3}\right]$.

When the osmotic pressures $\Pi_{m i x}$ and $\Pi_{e l}$ are equal, the microgel is in thermodynamic equilibrium. By combining Eqs. (9) and (12), the expression for equilibrium is obtained and the hydrodynamic radius of the particle $R_{\mathrm{H}}$ is given by the Flory-Rehner equation:

$$
\begin{aligned}
& \ln \left[1-\phi_{0}\left(\frac{R_{\mathrm{H}, 0}}{R_{\mathrm{H}}}\right)^{3}\right]+\phi_{0}\left(\frac{R_{\mathrm{H}, 0}}{R_{\mathrm{H}}}\right)^{3}+\chi \phi_{0}^{2}\left(\frac{R_{\mathrm{H}, 0}}{R_{\mathrm{H}}}\right)^{6} \\
& +\frac{\phi_{0}}{N_{G e l}}\left[\frac{R_{\mathrm{H}, 0}}{R_{\mathrm{H}}}-\frac{1}{2}\left(\frac{R_{\mathrm{H}, 0}}{R_{\mathrm{H}}}\right)^{3}\right]=0
\end{aligned}
$$

where $N_{G e l}$ is the average degree of polymerization of a polymer chain or the number of segments between two crosslinking points. The hydrodynamic radius of the particle $R_{\mathrm{H}}$ is a free variable, which can be found by solving Eq. (13).

\section{Interaction parameter}

The interaction parameter $\chi$ describes the affinity between the polymer and the solvent. Below the VPTT, polymer-solvent contacts are preferred compared to solvent-solvent contacts. As a result, the microgel is in the swollen state. In this case $\chi$ is less than 0.5. Above the VPTT, solvent-solvent contacts and polymer-polymer contacts are preferred. The microgel is in the collapsed state. In this case $\chi$ is larger than 0.5. Empirically, it has been found that $\chi$ can be modeled by various functions.

\section{Original}

In the original works, the Flory-Huggins parameter $\chi$ is given by [22]:

$\chi=\frac{\Delta H_{S P}-T \Delta S_{S P}}{\mathrm{k}_{\mathrm{B}} T}=\frac{1}{2}-A\left(1-\frac{\Theta}{T}\right)$

where $\Delta H_{S P}$ and $\Delta S_{S P}$ are the changes in enthalpy and entropy when a solvent-polymer contact occurs. $T$ is the thermodynamic temperature, $A=\left(2 \Delta S_{S P}+\mathrm{k}_{\mathrm{B}}\right) / 2 \mathrm{k}_{\mathrm{B}}$ is the dimensionless parameter, $\Theta=2 \Delta H_{S P} /\left(2 \Delta S_{S P}+\mathrm{k}_{\mathrm{B}}\right)$ is the characteristic temperature of the volume phase transition ( $\Theta$ temperature). To our knowledge, in most cases Eq. (14) alone could not be successfully applied to describe the swelling behavior of microgels. For the calculation of $\chi$, the interactions only with the nearest neighbor are considered.

\section{Series expansion}

Erman and Flory concluded on the basis of a very high packing density of the microgel, that higher-order interactions must also be considered. Therefore, they modeled the interaction parameter by a series expansion with respect to the volume fraction [30]:

$\chi=\chi_{1}+\chi_{2} \phi+\chi_{3} \phi^{2}+\ldots$

with $\chi_{1}=\frac{1}{2}-A\left(1-\frac{\Theta}{T}\right)$. With this approach, the swelling behavior of many microgels has been successfully described. But the original Flory-Rehner theory does not take into account that the cross-linking leads to a cooperativity of the VPT. The observed cooperativity of the VPT can be explained by the inhomogeneous distribution of chain length between two cross-linkers since the lower critical solution temperature (LCST) of chains with different lengths is not equal [33]. When a single chain crumples, collapse is induced in the neighboring chains because the chains are mechanically connected. If the chain lengths were homogeneously distributed, a discontinuous volume phase transition would be expected since all chains would collapse simultaneously[33] . 


\section{Hill-Like equation}

To account for the cooperativity of the volume phase transition, Leite et al. and Friesen et al. [31, 32] proposed a Hill-like model for the interaction parameter $\chi$ that yields physically meaningful parameters [Eq. (16)].

$\chi(t)=\chi_{0}+a\left(t-t_{a}\right)+b \frac{t_{r e l}^{v}}{t_{r e l}^{v}+K}$

where $\chi_{0}$ is the value of the $\chi$ parameter at $t=t_{a}, t_{a}$ is the first temperature data point, and $t_{e}$ is the last (end) temperature point of the data set, $a$ is the slope of the baseline, $b$ is the dimensionless amplitude parameter of the Hill transition, $K$ is the half-saturation constant, $v$ is the Hill parameter, and $t_{\text {rel }}(t)=\frac{t-t_{a}}{t_{e}-t_{a}}$ is the relative temperature. The relative temperature $t_{r e l}(t)$ is used analogously to the polymer concentration [32]. The Hill-like equation describes the cooperativity of the binding of water by the polymer. The state before and after VPT is described by the reaction:

$\mathrm{PS}_{v} \rightleftarrows \mathrm{P}+v \mathrm{~S}$.

where $v$ is the stoichiometric coefficient of the reaction, $P$ and $\nu S$ denote the states of the polymer and solvent after the VPT, respectively. The symbol $P S_{v}$ denotes the aggregate state after the VPT. Similar to the lyotropic transition, in the thermotropic transition the concentration of the species is replaced by a relative temperature $t_{r e l}$ that changes in the range $0 \leq t_{\text {rel }}(t) \leq 1$.

\section{Materials and methods}

\section{Chemicals}

$\mathrm{N}$-isopropyl-acrylamide (Sigma-Aldrich Chemie $\mathrm{GmbH}$, Munich, Germany; purity 97\%) was purified by recrystallization from hexane. The cross-linker $N, N$ '-methylenebisacrylamide (Sigma-Aldrich Chemie GmbH, Munich, Germany; purity 99\%) and the initiator ammonium persulfate (APS; Sigma-Aldrich Chemie GmbH, Munich, Germany; purity $\geq 98 \%$ ), were used without further purification. For all experiments, purified water from an Arium pro VF system (Sartorius AG, Göttingen, Germany) was used.

\section{Synthesis of the homopolymer microgels}

The homopolymer microgels of NIPAM were synthesized via conventional precipitation polymerization without surfactant. All syntheses are performed in $250 \mathrm{~mL}$ three-neck flasks equipped with a reflux condenser, mechanical stirrer $(210 \mathrm{rpm})$, and a nitrogen inlet. The monomer $(11.05 \mathrm{mmol})$ and the cross-linker $\mathrm{N}, \mathrm{N}$-methylenebisacrylamide $(5.0 \mathrm{~mol} \%$,
$10.0 \mathrm{~mol} \%, 15.0 \mathrm{~mol} \%$ with respect to the total monomer amount) are dissolved in $150 \mathrm{~mL}$ purified water and heated to $70{ }^{\circ} \mathrm{C}$ under continuous stirring and purged with nitrogen for $1 \mathrm{~h}$. Subsequently, the polymerization is initiated by the addition of $2 \mathrm{~mL}$ of the $0.2 \mathrm{M}$ solution of APS and left to proceed for $4 \mathrm{~h}$ at $70{ }^{\circ} \mathrm{C}$. Subsequently, the solution is cooled to room temperature and stirred overnight. For purification, all samples are treated by four cycles of centrifugation, decantation, and redispersion in purified water using a JA-30.50 Ti Rotor in an Avanti J-30I centrifuge (Beckman Coulter, Brea, CA, USA) at $20,000 \mathrm{rpm}$ and $25^{\circ} \mathrm{C}$.

\section{Photon Correlation Spectroscopy (PCS)}

The PCS measurements were performed on a custom-built setup with a fixed scattering angle $\theta: 60^{\circ}$, utilizing a $\mathrm{He}-\mathrm{Ne}$ Laser (wavelength $\lambda=632.8 \mathrm{~nm}, 21 \mathrm{~mW}$, Thorlabs, Newton, MA, USA) and two photomultipliers (ALV/SO-SIPD, ALV$\mathrm{GmbH}$, Langen, Germany) in a pseudo-cross-correlation configuration. The signal was correlated with an ALV-6010 multiple-tau correlator (ALV-GmbH, Langen, Germany). Subsequently, the intensity-time correlation functions were converted to the field-time correlation function $g^{1}(t)$ and analyzed using the CONTIN software [34]. However, an analysis using a second-order cumulant function also leads to the same result within the experimental precision. The temperature was controlled via a thermostat (Phoenix II, Thermo Fisher Scientific, Waltham, MA, USA or a Haake C25P, Thermo Fisher Scientific, Waltham, MA, USA), and the sample was equilibrated for $25 \mathrm{~min}$ inside the decaline-filled refractive index matching bath. For each temperature, 5 consecutive measurements were performed. The obtained mean relaxation rates $\bar{\Gamma}$ of the $g^{1}(t)$ functions were converted to the hydrodynamic radius by:

$R_{\mathrm{H}}=\frac{k_{B} T}{6 \pi \eta \frac{\bar{\Gamma}}{q^{2}}}$

where $k_{B}$ is the Boltzmann constant, $\eta$ the solvent viscosity (water), $T$ the temperature in Kelvin, and $q=\frac{4 \pi n}{\lambda} \sin \frac{\theta}{2}$ the magnitude of the scattering vector. $n$ is the refractive index of the solvent.

\section{Results and discussion}

\section{Fits of the swelling curves with different approaches}

The hydrodynamic radius $R_{\mathrm{H}}$ as a function of temperature $t$ calculated using the Flory-Rehner Eq. (13) with different approaches [Eqs. (14), (15), and (16)] for the interaction parameter $\chi$ was fitted to the swelling curves of $\mathrm{p}$ (NIPAM) particles at different concentrations of BIS ( $5 \mathrm{~mol} \%, 10$ mol\% and $15.0 \mathrm{~mol} \%$ ), see Fig. 1 . The parameters $\phi_{0}, N_{G e l}$, 
$A$ and $\Theta$ were obtained as fitting parameters with the original approach; additionally for the series expansion to the first order the parameter $\chi_{2}$ and for the series expansion to the second order the parameter $\chi_{3}$, see Table 1 . The parameters $\phi_{0}, N_{G e l}, K$, and $v$ were obtained as fitting parameters for the approach with the Hill-like model; see Table 2.

To estimate the quality of the fits, $(c h i)^{2}$-values are calculated by [35]:

$(c h i)^{2}=\sum_{t_{a}}^{t_{e}} \frac{\left(R_{\mathrm{H}, \text { exp }}(t)-R_{\mathrm{H}, f i t}(t, \text { fit parameters })\right)^{2}}{R_{\mathrm{H}, f i t}(t, \text { fit parameters })}$

where $R_{\mathrm{H} \text { exp }}(t)$ is the experimentally determined curve and $R_{\mathrm{H}, f i t}(t$, fit parameters) is the fitted curve. The swelling curves were fitted almost perfectly well using the series expansion approach and the Hill-like model. The $(c h i)^{2}$-values of the fits with these approaches show comparable $(c h i)^{2}$-values. From the fits (Fig. 1), and from the $(c h i)^{2}$-values (Tables 1 and 2), it can be seen that the fits performed with the original approach are significantly poorer compared to the other approaches. However, the observation of the fit quality does not yet give information about the physical accuracy of the model.

\section{Discussion of the fit parameters}

To make a conclusion about the physical accuracy of the model, the computed fit parameters must have physically meaningful values.

\section{Parameter $\mathrm{A}$ and $\boldsymbol{\Theta}$ temperature}

The $\Theta$ temperature corresponds to the spinodal temperature of the VPT at which the polymer behaves like an ideal chain and the Flory parameter becomes $\chi_{1}=1 / 2$. The spinodal temperature was determined from the onset of the VPT, see Table 3. Here the $\Theta$ temperatures from the second-order approximation are closest to the spinodal temperature of the volume phase transition VPTT(onset), the $\Theta$ temperatures obtained from the first-order approximation deviate significantly from the spinodal temperature, see Table 3.

Using the parameters $\mathrm{A}$ and $\Theta$, the mixing entropy $\Delta S_{S P}$ and the mixing enthalpy $\Delta H_{S P}$ of a solvent molecule can be calculated:

$$
\begin{aligned}
& \Delta S_{S P}=\mathrm{k}_{\mathrm{B}}\left(A-\frac{1}{2}\right) \\
& \Delta H_{S P}=\Theta \frac{2 \Delta S_{S P}+\mathrm{k}_{\mathrm{B}}}{2} .
\end{aligned}
$$

The theoretical calculations show negative values for $\Delta S_{S P}$ and $\Delta H_{S P}$ which is expected for a polymer-solvent system with a LCST (Table 4.) Comparison of the theoretically determined values with differential scanning calorimetry (DSC) measurements [36, 37] shows that the values are larger by a factor of about two. The values of the original approach are even one order of magnitude too large. This deviation can probably be attributed to the rough approximations of the lattice model. However, they are consistent with other PCS-based studies in most cases [25, 28, 38-40]. Another criterion is the range in which the $\chi_{1}$ should lie. The $\chi_{1}$ calculated from $A$ and $\Theta$ should be between 0 and 1 in this temperature range $[26,28,38]$. Only for the second-order approximation the values for $\chi_{1}$ are almost all between 0 and 1 as expected, see Fig. 2. The other approaches show a significant number of values outside this range.

\section{Coefficients $\chi_{2}$ and $\chi_{3}$}

The coefficients of the first- and second-order approximation are purely empirical parameters for which the physical sense is not clear since they cannot be traced back to special interactions $[25,28,30]$. But based on the coefficient $\chi_{2}$ it can be estimated how far the gel is from the critical point. The theoretical threshold for a gel with infinitely long chains is $\chi_{2}=1 / 3$ [30]. If the value for a gel with infinitely long chains is larger than $1 / 3$ then the volume phase transition is discontinuous. Below this threshold, the transition is continuous. The threshold above which a discontinuous transition is observed increases with crosslinker concentration since an unusually strong concentration dependence of $\chi$ is required [30, 38, 40]. From the swelling curves, it is evident that the degree of continuity increases with cross-linker (BIS) concentration. The higher the BIS concentration, the larger the distance of the gel from the critical point. Therefore, there could be the expectation that $\chi_{2}$ would decrease with the increase in cross-linker. However, as the BIS concentration increases, so does the threshold for $\chi_{2}$ at which the gel reaches the critical point. It is difficult to expect a trend of $\chi_{2}$ since even if $\chi_{2}$ remained constant for all BIS concentrations, the gel would drift further away from the critical point with the increase in BIS concentration since the threshold of $\chi_{2}$ also increases. The threshold values for the gels with the BIS concentrations studied here are not known at least to our knowledge. Hence, it is impossible to interpret the trend of the coefficients in a correct way at this point.

However, the values obtained from the fits for $\chi_{2}$ and $\chi_{3}$ are consistent with many other works [39]. 

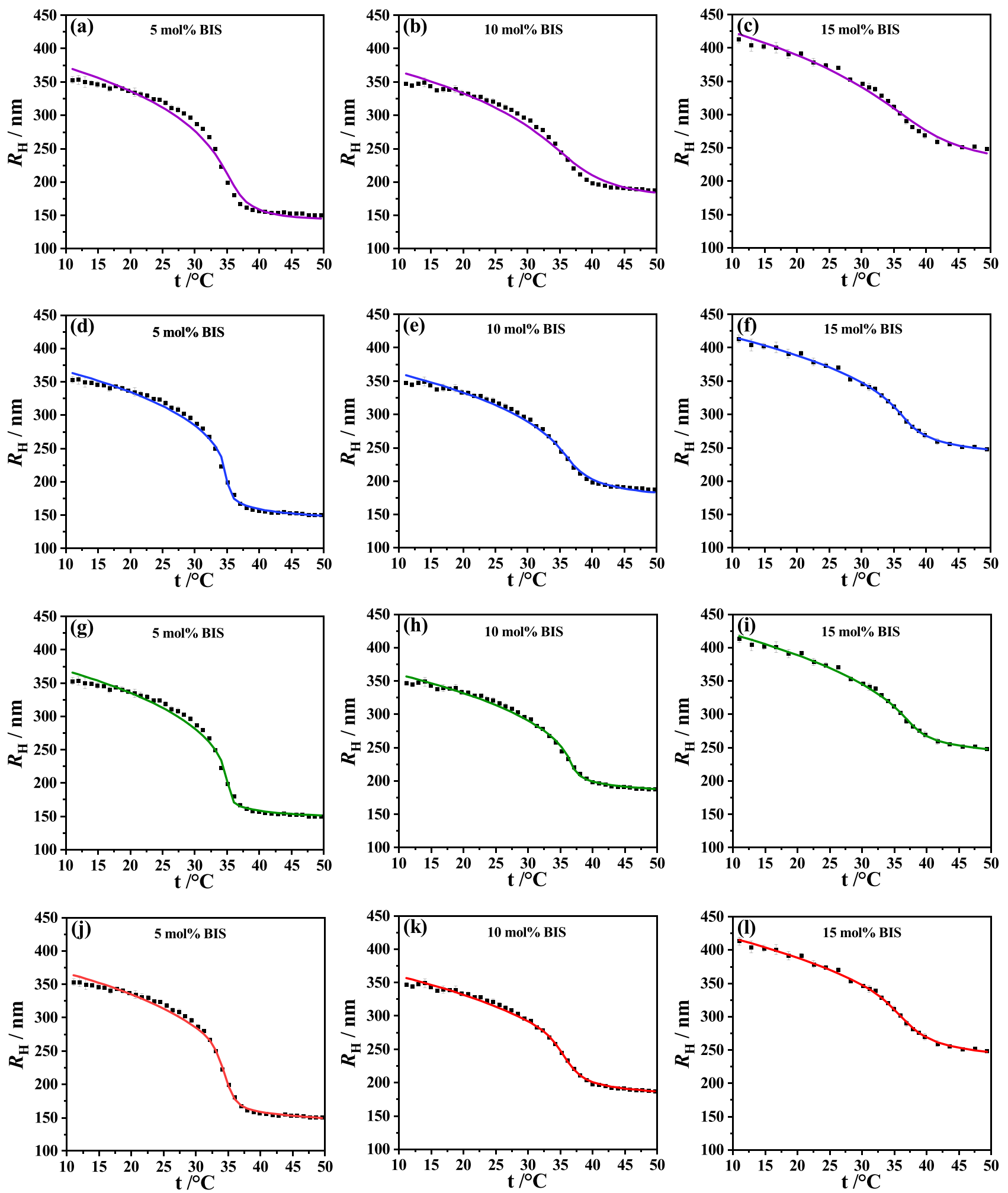

Fig. 1 Hydrodynamic radius $R_{\mathrm{H}}$ vs. temperature $t$ at different concentrations of the cross-linker BIS in p(NIPAM) particles. Squares are experimental data, solid curves represent the fitting curves. The fit of the hydrodynamic radii $R_{\mathrm{H}}$ (solid curve) was calculated using the Flory-Rehner Eq. (13). The interaction parameter $\chi$ of the FloryRehner Equation was calculated with different approaches: original approach [Eq. (14), (a-c)]; series expansion to the first order [Eq. (15), (d-f)]; series expansion to the second order [Eq. (15), (g-i)]; Hill-like Eq. (16), (j-l). A nearly perfect fit to the experimental data was achieved for the approach with the series expansions and with the Hill-like model 
Table 1 Parameters resulting from the fit of swelling curves with the original approach [Eq. (14)], and the series expansion to the first and second order [Eq. (15)] and the corresponding $(\text { chi })^{2}$-values [Eq. (19)] of the fits

\begin{tabular}{lcccccccc}
\hline & {$[\mathbf{B I S}] / \mathbf{m o l} \%$} & $\boldsymbol{N}_{\text {Gel }}$ & $\boldsymbol{\phi}_{0}$ & $\mathbf{A}$ & $\chi_{2}$ & $\chi_{3}$ & $\boldsymbol{\Theta} / \mathbf{K}$ & $(\boldsymbol{c h i})^{\mathbf{2}} / \mathbf{n m}$ \\
\hline Original & 5.00 & 32.50 & 0.84 & -35.2 & & & 308.3 & 12.49 \\
& 10.0 & 22.90 & 0.74 & -15.8 & & & 308.4 & 8.690 \\
First order & 15.0 & 13.40 & 0.68 & -13.6 & & & 309.5 & 2.950 \\
& 5.00 & 155.5 & 0.77 & -6.3 & 0.68 & & 313.2 & 3.677 \\
& 10.0 & 53.50 & 0.73 & -4.5 & 0.78 & & 320.9 & 1.278 \\
Second order & 5.00 & 16.80 & 0.78 & -6.2 & 0.94 & & 325.3 & 0.348 \\
& 10.0 & 457.8 & 0.85 & -6.3 & 0.33 & 0.54 & 310.4 & 5.050 \\
& 15.0 & 31.30 & 0.81 & -4.0 & 0.02 & 0.91 & 312.0 & 0.512 \\
\hline
\end{tabular}

Table 2 Parameters resulting from the fit of swelling curves with the Hill-like Eq. (16) and the corresponding $(\text { chi })^{2}$-values [Eq. (19)] of the fits

\begin{tabular}{lccccccc}
\hline & {$[\mathrm{BIS}] \mathbf{m o l} \%$} & $\boldsymbol{N}_{\boldsymbol{G e l}}$ & $\boldsymbol{\phi}_{\mathbf{0}}$ & $\boldsymbol{K} / \mathbf{1 0}^{-\mathbf{4}}$ & $\boldsymbol{v}$ & $\boldsymbol{t}_{\mathbf{0 . 5}} /{ }^{\circ} \mathrm{C}$ & $(\boldsymbol{c h i})^{\mathbf{2}} / \mathbf{n m}$ \\
\hline Hill-like & 5.00 & 213.8 & 0.72 & 0.307 & 22.7 & 35.4 & 2.639 \\
& 10.0 & 57.40 & 0.72 & 20.00 & 15.6 & 36.8 & 1.465 \\
& 15.0 & 27.50 & 0.72 & 230.0 & 10.5 & 37.8 & 0.436 \\
\hline
\end{tabular}

Table $3 \Theta$ temperatures resulting from the fit of swelling curves with the original approach [Eq. (14)], and the series expansion to the first and second order [Eq. (15)] and the spinodal temperatures determined by the onset of the VPT

\begin{tabular}{lllll}
\hline$[\mathbf{B I S}] / \mathbf{m o l} \%$ & $\begin{array}{l}\text { Original } \\
\boldsymbol{\Theta} / \mathrm{K}\end{array}$ & $\begin{array}{l}\text { First order } \\
\boldsymbol{\Theta} / \mathrm{K}\end{array}$ & $\begin{array}{l}\text { Second order } \\
\boldsymbol{\Theta} / \mathrm{K}\end{array}$ & 309.9 \\
\hline 5.00 & 308.3 & 313.2 & 310.4 & 312.7 \\
10.0 & 308.4 & 320.9 & 312.7 & 313.6 \\
15.0 & 309.5 & 325.3 & 312.0 & VPT(onset)/K \\
\hline
\end{tabular}

\section{Average degree of polymerization $N_{G e l}$}

If $N_{G e l}$ is homogeneously distributed in the gel and the cross-linker is fully incorporated, $N_{G e l}$ can be calculated by Eq. (22) [32].

$N_{\text {Gel }}=\frac{50 \mathrm{~mol} \%}{[\mathrm{BIS}]}$.
The theoretical values for $N_{G e l}$ are 10,5 and 3.3 for the p(NIPAM) microgels with $5 \mathrm{~mol} \%, 10 \mathrm{~mol} \%$ and $15 \mathrm{~mol} \%$ BIS, respectively. As expected, the values for $N_{G e l}$ obtained from the fits decrease in all cases with the increase in BIS (Tables 1 and 2). However, the values from the fits are all systematically larger than the theoretically calculated values for $N_{G e l}$. These values for $N_{G e l}$ are nevertheless consistent

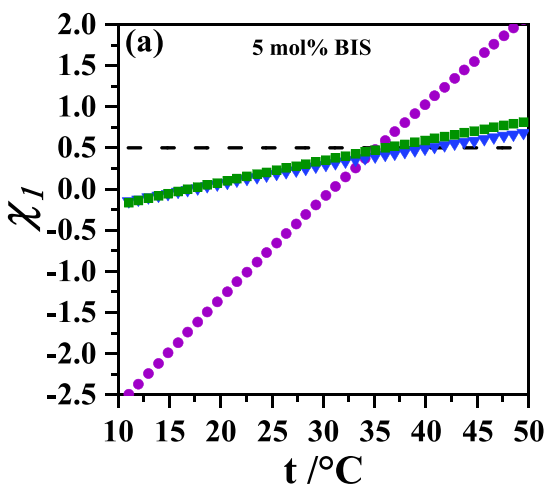

Fig. $2 \chi_{1}$ vs. temperature $t$ at different concentrations of BIS in $\mathrm{p}$ (NIPAM) particles; [BIS] $/ \mathrm{mol} \%=5.0(\mathbf{a}), 10.0(\mathbf{b}), 15.0$ (c). $\chi_{1}$ has been calculated with Eq. (14) from the different approaches; origi-
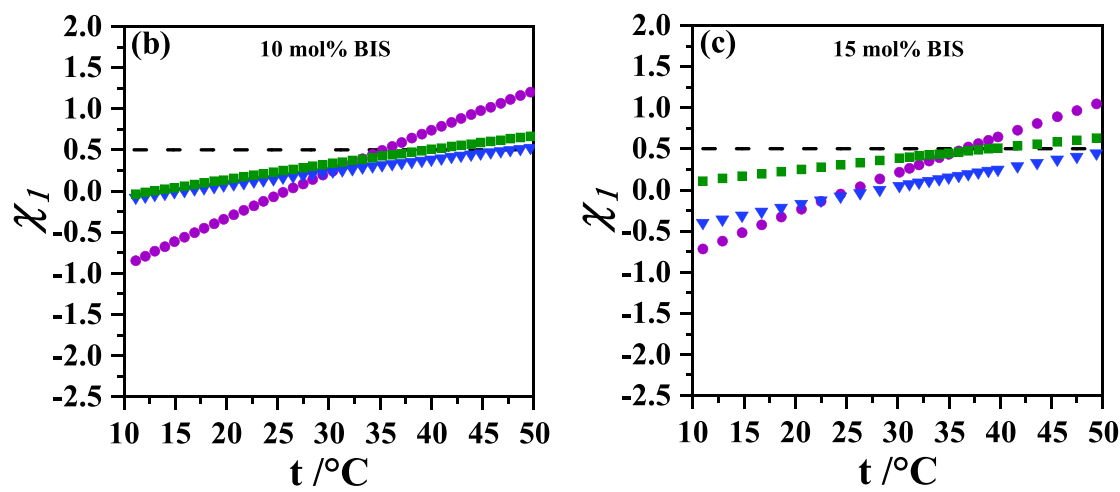

nal (circles), first order (triangles) and second order (squares). The dashed line indicates the value for $\chi_{1}=1 / 2$ at which the volume phase transition occurs 
Table 4 Theoretically calculated values for $\Delta S_{S P}$ and $\Delta H_{S P}$ using Eqs. (20) and (21)

\begin{tabular}{|c|c|c|c|c|c|c|}
\hline \multirow[b]{2}{*}{$\begin{array}{l}\text { [BIS] } \\
/ \mathrm{mol} \%\end{array}$} & \multicolumn{2}{|l|}{ Original } & \multicolumn{2}{|c|}{ First order } & \multicolumn{2}{|c|}{ Second order } \\
\hline & $\begin{array}{l}\Delta H_{S P} \\
/ 10^{-20} \mathrm{~J}\end{array}$ & $\begin{array}{l}\Delta S_{S P} \\
/ 10^{-23} \mathrm{~J} \mathrm{~K}^{-1}\end{array}$ & $\begin{array}{l}\Delta H_{S P} \\
/ 10^{-20} \mathrm{~J}\end{array}$ & $\begin{array}{l}\Delta S_{S P} \\
/ 10^{-23} \mathrm{~J} \mathrm{~K}^{-1}\end{array}$ & $\begin{array}{l}\Delta H_{S P} \\
/ 10^{-20} \mathrm{~J}\end{array}$ & $\begin{array}{l}\Delta S_{S P} \\
/ \mathbf{1 0}^{-\mathbf{2 3}} \mathbf{J ~ K}^{-1}\end{array}$ \\
\hline 5.00 & -10.8 & -35.7 & -2.73 & -9.42 & -2.69 & -9.37 \\
\hline 10.0 & -6.74 & -22.5 & -1.99 & -6.88 & -2.31 & -8.09 \\
\hline 15.0 & -5.80 & -19.4 & -2.78 & -9.24 & -1.74 & -6.29 \\
\hline
\end{tabular}

Table 5 The half-saturation temperature $t_{0.5}$ and the VPTT determined by the inflection point (IP) of the $R_{\mathrm{H}}(T)$-curves at different concentrations of the cross-linker BIS in p(NIPAM) particles

\begin{tabular}{lll}
\hline & Hill-like model & \\
{$[$ BIS] $/ \mathbf{m o l} \%$} & $t_{0.5} /{ }^{\circ} \mathrm{C}$ & VPTT $(I P) /{ }^{\circ} \mathrm{C}$ \\
\hline 5.00 & 35.4 & 34.1 \\
10.0 & 36.8 & 35.6 \\
15.0 & 37.8 & 36.3 \\
\hline
\end{tabular}

with the results of other $R_{\mathrm{H}^{-}}$-based studies [41, 42]. One reason for the systematical deviation could be the inhomogeneous distribution of $N_{G e l}$. This was also observed, e.g., in super-resolution fluorescence microscopy measurements [43].

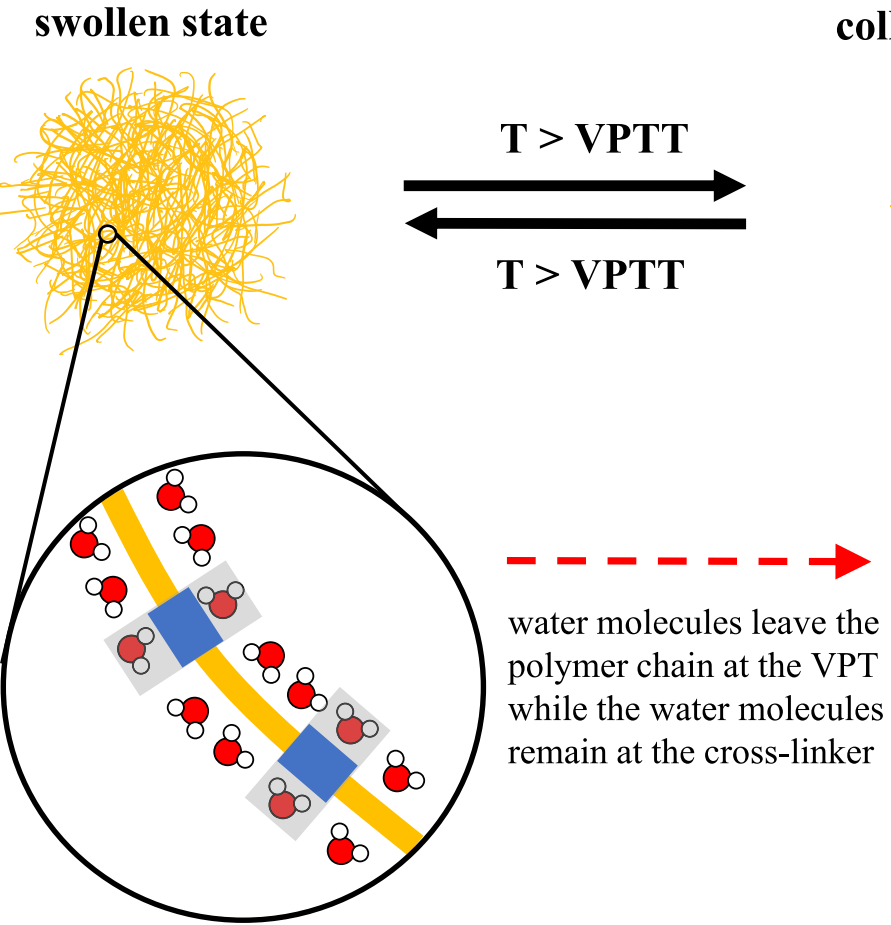

Fig. 3 A schematic representation of the volume phase transition. It is assumed that the water molecules bound to the monomer units leave the segment cooperatively at the volume phase transition while

\section{Polymer volume fraction in the reference state $\phi_{0}$}

The polymer volume fraction $\boldsymbol{\phi}_{0}$ obtained from the fits of the swelling curves with the Hill-like model is 0.72 for all concentrations of BIS (Table 2). The $\phi_{0}$ obtained with the original approach decreases with BIS concentration from 0.84 to 0.68. A similar trend is also observed with the second-order approximation approach where the values for $\phi_{0}$ decrease from 0.85 to 0.81 , while with the first-order approximation the values show no trend and are between 0.73 and 0.78 (Table 1). These values for $\phi_{0}$ are consistent with the results of other $R_{\mathrm{H}}$-based studies [39, 41, 44, 45]. However, the values from $R_{\mathrm{H}}$-based studies are in contradiction with the results of $\phi_{0}=0.4-0.6$ obtained by small-angle neutronscattering (SANS) $[39,46,47]$. The difference might be due collapsed state

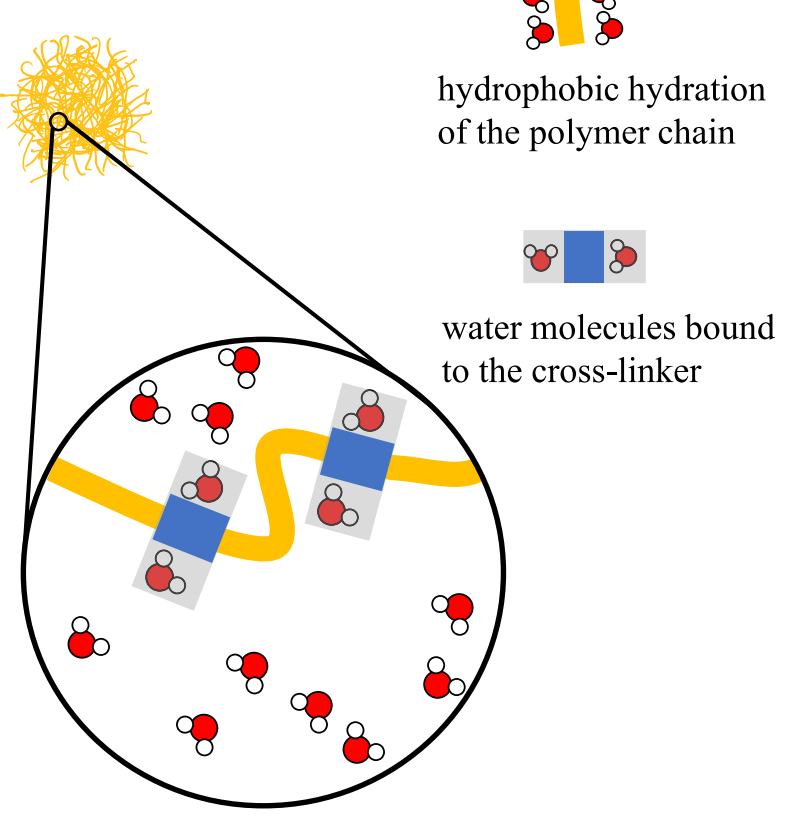

the water molecules bound to the cross-linker remain at their position because the cross-linker has no lower critical solution temperature 


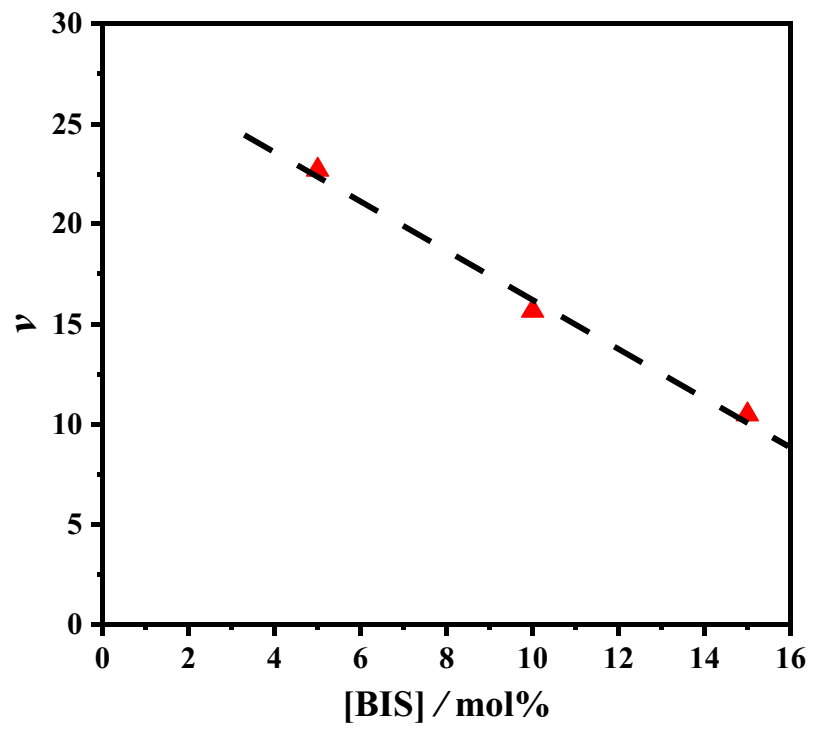

Fig. 4 The Hill parameter $v$ vs. concentration of BIS in p(NIPAM) particles. The Hill parameter has been calculated with the Hill-like Eq. (16). The dashed line is a guide for the eye

to the different weighting of the contribution of the outer fuzzy regions in SANS compared to PCS. Furthermore, for a direct comparison, the $p(r)$ density functions obtained from the SANS measurements must be integrated over the whole particle radius $[32,46]$.

\section{Half-saturation Constant $K$}

The half-saturation constant $K$ of the Hill-like model is given by [32]:

$K=\left(\frac{t_{0.5}-t_{a}}{t_{e}-t_{a}}\right)^{v}$.

$K$ corresponds to the concentration of segments which are still swollen with water at the half-saturation temperature $t_{0.5}$. This concentration is equal to the concentration of segments that are already collapsed or at least dehydrated at $t_{0.5}$ [32]. We assume that at the inflection point of the VPT, half of the water molecules have left the gel compared to the total number molecules which leave the gel when the transition is complete. Therefore, $t_{0.5}$ would be expected to correspond to the VPTT determined at the inflection point of the $R_{\mathrm{H}}(T)$ -curves. As expected, in all cases $t_{0.5}$ is approximately equal to the VPTT (Table 5).

\section{Hill parameter $v$}

The Hill parameter $v$ linearly decreases with the increase in the cross-linker concentration BIS, see Fig. 4 [32]. We assume that $v$ represents the number of water molecules per segment of a certain length that cooperatively leave the segment at the VPT. Since the cross-linker has no LCST, the water molecules attached to the cross-linker should not leave the segment at the VPT (Fig. 3). The linear decrease in $v$ with the increase in [BIS] confirms this assumption that only water molecules attached to the monomer units cooperatively leave the segment, see Fig. 4 [32]. The small values for the number of water molecules per segment are in qualitative agreement with results from recent molecular dynamics simulations [48, 49].

\section{Conclusions}

In the present work, we compared different approaches which are used to describe the polymer-solvent interaction in thermoresponsive microgels at different cross-linker concentrations.

The work reveals that the Hill-like model for the description of the swelling behavior provides physically reasonable and meaningful parameters in contrast to the other approaches. The original approach of Flory and Huggins for the polymer-solvent interaction parameter could not successfully describe the data. The series expansion up to first order could describe the data well but some parameters obtained from the fits are physically not meaningful, for instance, the large deviation of the $\Theta$ temperatures from the spinodal temperatures. The series expansion up to second order could also describe the data well. The $\Theta$ temperatures also are in good accordance with the spinodal temperatures of the volume phase transition but the values for $\Delta S_{S P}$ and $\Delta H_{S P}$ are larger by a factor of 2 compared to differential scanning calorimetry (DSC) measurements [36, 37]. It is also not clear which physical meaning the fit parameters $\chi_{1}$ and $\chi_{2}$ have. Furthermore, the original approach of Flory and Huggins and the series expansion do not take into account the cooperativity of the volume phase transition. It is obvious that the volume phase transition must be cooperative since all meshes of the gel network are mechanically coupled.

The Hill model, which takes into account the cooperativity of the volume phase transition, describes the data well and also provides physically reasonable parameters. Thus, the half-saturation temperature $t_{0.5}$ calculated from the fit parameter $K$ corresponds as expected to the volume phase transition temperature. Moreover, ${ }_{v}$ corresponds to the number of water molecules cooperatively leaving the gel per segment during the volume phase transition. The small number of water molecules found here at least qualitatively agrees with molecular dynamic simulations. [48, 49] Furthermore, a linear dependence of $v$ with [BIS] was observed which is consistent with the assumption that water molecules attached to the cross-linker do not leave the gel at the volume phase transition since BIS does not possess a LCST [32]. 
Acknowledgements We acknowledge support for the publication costs by the Open Access Publication Fund of Bielefeld University.

Funding Open Access funding enabled and organized by Projekt DEAL.

\section{Declarations}

Conflicts of interest The authors declare no conflict of interest.

Open Access This article is licensed under a Creative Commons Attribution 4.0 International License, which permits use, sharing, adaptation, distribution and reproduction in any medium or format, as long as you give appropriate credit to the original author(s) and the source, provide a link to the Creative Commons licence, and indicate if changes were made. The images or other third party material in this article are included in the article's Creative Commons licence, unless indicated otherwise in a credit line to the material. If material is not included in the article's Creative Commons licence and your intended use is not permitted by statutory regulation or exceeds the permitted use, you will need to obtain permission directly from the copyright holder. To view a copy of this licence, visit http://creativecommons.org/licenses/by/4.0/.

\section{References}

1. Karg M et al (2019) Nanogels and microgels: From model colloids to applications, recent developments, and future trends. Langmuir 35(19):6231-6255. https://doi.org/10.1021/acs.langmuir.8b04304

2. Pelton RH, Chibante P (1986) Preparation of aqueous latices with N-isopropylacrylamide. Colloids Surf 20(3):247-256. https://doi. org/10.1016/0166-6622(86)80274-8

3. Pelton R (2000) Temperature-sensitive aqueous microgels. Adv Colloid Interf Sci 85(1):1-33. https://doi.org/10.1016/S00018686(99)00023-8

4. Thorne JB, Vine GJ, Snowden MJ (2011) Microgel applications and commercial considerations. Colloid Polym Sci 289(5-6):625646. https://doi.org/10.1007/s00396-010-2369-5

5. Saunders BR, Vincent B (1999) Microgel particles as model colloids: theory, properties and applications. Adv Colloid Interf Sci 80(1):1-25. https://doi.org/10.1016/S0001-8686(98)00071-2

6. Wedel B, Zeiser M, Hellweg T (2012) Non NIPAM based smart microgels: Systematic variation of the volume phase transition temperature by copolymerization. Z Phys Chem 226(7-8):737748. https://doi.org/10.1524/zpch.2012.0267

7. Nayak S, Lyon LA (2005) Weiche nanotechnologie mit weichen nanopartikeln. Angew Chem 117(47):7862-7886. https://doi.org/ 10.1002/ange.200501321

8. Plamper FA, Richtering W (2017) Functional microgels and microgel systems. Acc Chem Res 50(2):131-140. https://doi.org/ 10.1021/acs.accounts.6b00544

9. Richtering W, Saunders BR (2014) Gel architectures and their complexity. Soft Matter 10(21):3695-3702. https://doi.org/10. 1039/C4SM00208C

10. Pich A, Richtering W (eds) (2010) Chemical design of responsive microgels vol. 234 of Advances in Polymer Science. Springer, Heidelberg

11. Hannappel Y, Wiehemeier L, Dirksen M, Kottke T, Hellweg T (2021) Smart microgels from unconventional acrylamides. Macromol Chem Phys 222(13):2100067. https://doi.org/10.1002/macp. 202100067

12. Dirksen M, Kinder TA, Brändel T, Hellweg T (2021) Temperature controlled loading and release of the anti-inflammatory drug cannabidiol by smart microgels. Molecules (Basel, Switzerland) 26(11). https://doi.org/10.3390/molecules26113181

13. Langer R (1990) New methods of drug delivery. Science (New York, NY) 249(4976):1527-1533. https://doi.org/10.1126/science.2218494

14. Smeets NMB, Hoare T (2013) Designing responsive microgels for drug delivery applications. J Polym Sci A Polym Chem 51(14):3027-3043. https://doi.org/10.1002/pola.26707

15. Park TG, Hoffman AS (1990) Immobilization and characterization of beta-galactosidase in thermally reversible hydrogel beads. J Biomed Mater Res 24(1):21-38. https://doi.org/10. 1002/jbm.820240104

16. Welsch N, Becker AL, Dzubiella J, Ballauff M (2012) Coreshell microgels as "smart" carriers for enzymes. Soft Matter 8(5):1428-1436. https://doi.org/10.1039/C1SM06894F

17. Uhlig K, Wegener T, He J, Zeiser M, Bookhold J, Dewald I, Godino N, Jaeger M, Hellweg T, Fery A, Duschl C (2016) Patterned thermoresponsive microgel coatings for noninvasive processing of adherent cells. Biomacromolecules 17(3):1110-1116. https://doi.org/10.1021/acs.biomac.5b01728

18. Lu Y, Mei Y, Drechsler M, Ballauff M (2006) Thermosensitive core-shell particles as carriers for ag nanoparticles: modulating the catalytic activity by a phase transition in networks. Angewandte Chemie (International ed in English) 45(5):813-816. https://doi.org/10.1002/anie.200502731

19. Sabadasch V, Wiehemeier L, Kottke T, Hellweg T (2020) Coreshell microgels as thermoresponsive carriers for catalytic palladium nanoparticles. Soft Matter 16(23):5422-5430. https:// doi.org/10.1039/D0SM00433B

20. Lu Y, Spyra P, Mei Y, Ballauff M, Pich A (2007) Composite hydrogels: Robust carriers for catalytic nanoparticles. Macromol Chem Phys 208(3):254-261. https://doi.org/10.1002/macp. 200600534

21. Dušek K (1993) Responsive Gels: Volume Transitions I, Advances in Polymer Science, vol 109. Springer, Berlin and Heidelberg. https://doi.org/10.1007/3-540-56791-7

22. Flory PJ, Rehner J (1943) Statistical mechanics of cross-linked polymer networks i. rubberlike elasticity. J Chem Phys 11(11):512_ 520. https://doi.org/10.1063/1.1723791

23. Flory PJ (1953) Principles of polymer chemistry, 19th edn. Cornell Univ. Press, Ithaca, NY

24. Graessley WW (2004) Polymeric liquids and networks: Structure and properties. Garland Science, New York. http://www.loc.gov/ catdir/enhancements/fy0652/2003048324-d.html 14 Feb 2022.

25. Hirotsu S (1994) Static and time-dependent properties of polymer gels around the volume phase transition. Phase Transit 47(3-4):183-240. https://doi.org/10.1080/01411599408200347

26. Nigro V, Angelini R, Bertoldo M, Bruni F, Ricci MA, Ruzicka B (2017) Dynamical behavior of microgels of interpenetrated polymer networks. Soft Matter 13(30):5185-5193. https://doi. org/10.1039/c7sm00739f

27. Nigro V, Angelini R, Rosi B, Bertoldo M, Buratti E, Casciardi S, Sennato S, Ruzicka B (2019) Study of network composition in interpenetrating polymer networks of poly(N-isopropylacrylamide) microgels: The role of poly(acrylic acid). J Colloid Interface Sci 545:210-219. https://doi.org/10.1016/j.jcis.2019.03.004

28. López-León T, Fernández-Nieves A (2007) Macroscopically probing the entropic influence of ions: deswelling neutral microgels with salt. Phys Rev E Stat Nonlinear Soft Matter Phys 75(1 Pt 1):011801. https://doi.org/10.1103/PhysRevE.75.011801

29. Shibayama M, Shirotani Y, Hirose H, Nomura S (1997) Simple scaling rules on swollen and shrunken polymer gels. Macromolecules 30(23):7307-7312. https://doi.org/10.1021/ma970443w

30. Erman B, Flory PJ (1986) Critical phenomena and transitions in swollen polymer networks and in linear macromolecules. Macromolecules 19(9):2342-2353. https://doi.org/10.1021/ ma00163a003 
31. Leite DC, Kakorin S, Hertle Y, Hellweg T, da Silveira NP (2018) Smart starch-poly(N-isopropylacrylamide) hybrid microgels: Synthesis, structure, and swelling behavior. Langmuir 34(37):1094310954. https://doi.org/10.1021/acs.langmuir.8b00706

32. Friesen S, Hannappel Y, Kakorin S, Hellweg T (2021) Accounting for cooperativity in the thermotropic volume phase transition of smart microgels. Gels 7(2):42. https://doi.org/10.3390/ gels 7020042

33. Wu C, Zhou S (1997) Volume phase transition of swollen gels: Discontinuous or continuous? Macromolecules 30(3):574-576. https://doi.org/10.1021/ma960499a

34. Provencher SW (1982) Contin: a general purpose constrained regularization program for inverting noisy linear algebraic and integral equations. Comput Phys Commun 27(3):229-242. https:// doi.org/10.1016/0010-4655(82)90174-6

35. Press WH, Teukolsky SA, Vetterling WT, Flannery BP (2007) Numerical recipes: the art of scientific computing, 3rd edn. Cambridge University Press, Cambridge

36. Otake K, Inomata H, Konno M, Saito S (1990) Thermal analysis of the volume phase transition with $\mathrm{N}$-isopropylacrylamide gels. Macromolecules 23(1):283-289. https://doi.org/10.1021/ma00203a049

37. Gao Y, Yang J, Ding Y, Ye X (2014) Effect of urea on phase transition of poly( $\mathrm{N}$-isopropylacrylamide) investigated by differential scanning calorimetry. J Phys Chem B 118(31):9460-9466. https:// doi.org/10.1021/jp503834c

38. Fernández-Barbero A, Fernández-Nieves A, Grillo I, LópezCabarcos E (2002) Structural modifications in the swelling of inhomogeneous microgels by light and neutron scattering. Phys Rev E Stat Nonlinear Soft Matter Phys 66(5 Pt 1):051803. https:// doi.org/10.1103/PhysRevE.66.051803

39. Lopez CG, Richtering W (2017) Does Flory-Rehner theory quantitatively describe the swelling of thermoresponsive microgels? Soft Matter 13(44):8271-8280. https://doi.org/10.1039/c7sm01274h

40. Hirotsu S, Hirokawa Y, Tanaka T (1987) Volume-phase transitions of ionized N-isopropylacrylamide gels. J Chem Phys 87(2):13921395. https://doi.org/10.1063/1.453267

41. Crassous JJ, Wittemann A, Siebenbürger M, Schrinner M, Drechsler M, Ballauff M (2008) Direct imaging of temperaturesensitive core-shell latexes by cryogenic transmission electron microscopy. Colloid Polym Sci 286(6-7):805-812. https://doi. org/10.1007/s00396-008-1873-3
42. Karg, Matthias, Prévost, Sylvain, Brandt, Astrid, Wallacher, Dirk, von Klitzing, Regine and Hellweg, Thomas (2013) Poly-NIPAM microgels with different cross-linker densities. 63-76. https://doi. org/10.1007/978-3-319-01683-2_6

43. Bergmann S, Wrede O, Huser T, Hellweg T (2018) Super-resolution optical microscopy resolves network morphology of smart colloidal microgels. Phys Chem Chem Phys 20(7):5074-5083. https://doi. org/10.1039/C7CP07648G

44. Sierra-Martín B, Choi Y, Romero-Cano MS, Cosgrove T, Vincent B, Fernández-Barbero A (2005) Microscopic signature of a microgel volume phase transition. Macromolecules 38(26):1078210787. https://doi.org/10.1021/ma0510284

45. Voudouris P, Florea D, van der Schoot P, Wyss HM (2013) Micromechanics of temperature sensitive microgels: dip in the Poisson ratio near the LCST. Soft Matter 9(29):7158. https://doi.org/10. 1039/C3SM50917F

46. Stieger M, Richtering W, Pedersen JS, Lindner P (2004) Smallangle neutron scattering study of structural changes in temperature sensitive microgel colloids. J Chem Phys 120(13):6197-6206. https://doi.org/10.1063/1.1665752

47. Cors M, Wiehemeier L, Hertle Y, Feoktystov A, Cousin F, Hellweg $\mathrm{T}$, Oberdisse J (2018) Determination of internal density profiles of smart acrylamide-based microgels by small-angle neutron scattering: A multishell reverse monte carlo approach. Langmuir 34(50):1540315415. https://doi.org/10.1021/acs.langmuir.8b03217

48. Tavagnacco L, Zaccarelli E, Chiessi E (2018) On the molecular origin of the cooperative coil-to-globule transition of poly $(\mathrm{N}$ isopropylacrylamide) in water. Phys Chem Chem Phys 20(15):999710010. https://doi.org/10.1039/C8CP00537K

49. Chiessi E, Paradossi G (2016) Influence of tacticity on hydrophobicity of poly( $\mathrm{N}$-isopropylacrylamide): a single chain molecular dynamics simulation study. J Phys Chem B 120(15):3765-3776. https://doi.org/10.1021/acs.jpcb.6b01339

Publisher's Note Springer Nature remains neutral with regard to jurisdictional claims in published maps and institutional affiliations. 